On the structural, electronic and magnetic properties of $\mathrm{MnCr}_{2} \mathrm{O}_{4}$ spinel

This content has been downloaded from IOPscience. Please scroll down to see the full text. 1997 J. Phys.: Condens. Matter 910715

(http://iopscience.iop.org/0953-8984/9/48/014)

View the table of contents for this issue, or go to the journal homepage for more

Download details:

IP Address: 193.54.110.42

This content was downloaded on 23/05/2015 at 13:40

Please note that terms and conditions apply. 


\title{
On the structural, electronic and magnetic properties of $\mathrm{MnCr}_{2} \mathrm{O}_{4}$ spinel
}

\author{
F Freyria Fava $\nmid$, I Baraille $\ddagger$, A Lichanot $\ddagger$, C Larrieuł and R Dovesi $\dagger$ \\ $\dagger$ Department CIFM, University of Torino, via Giuria 5, I-10125 Torino, Italy \\ $\ddagger$ Laboratoire de Chimie Structurale, UMR 5624, IFR rue Jules Ferry, 64000 Pau, France
}

Received 11 July 1997

\begin{abstract}
The structural, electronic and magnetic properties of $\mathrm{MnCr}_{2} \mathrm{O}_{4}$ spinel are investigated using the periodic ab initio Hartree-Fock program CRYSTAL. The geometry is fully optimized and the bulk modulus evaluated. The system is a large-gap insulator; the $\mathrm{Mn}-\mathrm{O}$ bond is fully ionic, whereas in the $\mathrm{Cr}-\mathrm{O}$ case there is a relatively large overlap between the $\mathrm{Cr} \mathrm{d}$ and the $\mathrm{O}$ sp orbitals, and the resulting bond has some covalent character. The ferromagnetic solution (all spins in the same direction) and two ferrimagnetic solutions (one or two $\mathrm{Mn}$ atoms of the unit cell with spin down, the remaining transition metal atoms with spin up) have been considered; the latter are more stable than the former by 2.2 and 3.8 mHartree per unit cell, respectively. At zero pressure, the spinel is more stable than the mixture of simple oxides $\mathrm{MnO}+\alpha-\mathrm{Cr}_{2} \mathrm{O}_{3}$ by about $17 \mathrm{kcal} \mathrm{mol}^{-1}$, in excellent agreement with experiment.
\end{abstract}

\section{Introduction}

Powerful computational tools have been made available in the past few years for simulating $a b$ initio ground-state properties of crystalline solids containing transition metal salts and/or having relatively large unit cells. The periodic Hartree-Fock scheme [1, 2], in particular, has been shown to be able to reproduce qualitatively and semi-quantitatively not only the structural and electronic properties of simple transition metal oxides such as $\mathrm{MnO}$ and $\mathrm{NiO}[3,4]$, and of relatively complex structures such as $\mathrm{Fe}_{2} \mathrm{O}_{3}$ [5] and $\mathrm{Cr}_{2} \mathrm{O}_{3}$ [6], but also more delicate quantities such as the relative stability of different magnetic phases, in spite of the limitation related to the neglect of electron correlation. Recent improvements of the CRYSTAL95 computer code [7], both in terms of speed and of tools for addressing the required magnetic solution and for controlling the self-consistent-field process when many transition metal ions with open-shell structure are present in the unit cell, now permit the investigation of relatively complicated structures with more than one type of transition metal atom. The spinels $\mathrm{AB}_{2} \mathrm{O}_{4}$ [8] represent one of the most important and interesting families of crystalline compounds, with applications in many different areas. The present method has been applied in the past to the study of one of the simplest members of the family, namely $\mathrm{MgAl}_{2} \mathrm{O}_{4}$ [9]. The structural and elastic properties of this compound have been correctly reproduced, as well as its relative stability with respect to the simple oxides $\mathrm{MgO}$ and $\alpha-\mathrm{Al}_{2} \mathrm{O}_{3}$. In the present paper, we investigate for the first time the structural, electronic and magnetic properties of a spinel with two different transition metal atoms, namely $\mathrm{MnCr}_{2} \mathrm{O}_{4}$. The structure of $\mathrm{MnCr}_{2} \mathrm{O}_{4}$, which contains two formula units per cell (14 atoms), has been optimized and the bulk modulus evaluated; the electronic properties will be discussed in terms of Mulliken population analysis data, band structures, densities 
of states, and charge- and spin-density maps. The stability of the spinel with respect to $\mathrm{MnO}$ and $\alpha-\mathrm{Cr}_{2} \mathrm{O}_{3}$ is calculated. As regards the magnetic properties, three structures have been considered which do not require large unit cells or a substantial symmetry reduction: the ferromagnetic system (FEM), in which all spins of the uncoupled electrons are up, and two ferrimagnetic solutions, with spins down for one $\left(\mathrm{FIM}_{1}\right)$ or two $\left(\mathrm{FIM}_{2}\right) \mathrm{Mn}$ atoms in the unit cell (all $\mathrm{Cr}$ atoms having spin up).

\section{Computational details}

For the present calculations, the CRYSTAL95 computer program [7] has been used, which contains an unrestricted Hartree-Fock (UHF) option for the treatment of spin-polarized systems. This feature is necessary for obtaining spin-polarized eigenfunctions of the Fock Hamiltonian, because of the presence of unpaired 3d electrons on $\mathrm{Cr}$ and $\mathrm{Mn}$. We refer the reader to previous papers $[1,2]$ for a description of the periodic Hartree-Fock crystalline-orbitals LCAO self-consistent-field computational scheme as implemented in the CRYSTAL95 code [7]; the UHF formulation for periodic systems and applications to transition metal compounds can be found in references [6-10]. Tools have been implemented in CRYSTAL95 in order to direct the system towards the required spin solution. It is, however, to be remembered that only the total number of unpaired electrons in the unit cell is imposed a priori, whereas no constraint at all is imposed on the partition of the spin density between the transition metal atoms (four $\mathrm{Cr}$ and two $\mathrm{Mn}$ in the present case), and among the $\mathrm{d}$ orbitals of a given atom. The final spin configuration in the unit cell is then the result of a variational calculation, with the constraint of the number of $\alpha-\beta$ electrons being defined a priori. As regards the computational conditions, the numerical values of the tolerance parameters involved in the evaluation of the infinite bielectronic Coulomb and exchange series were identical to those adopted in recent studies [3-6], and were chosen to ensure high numerical accuracy. The shrinking factor, $S$, defining the reciprocal space net is 4 , corresponding to eight reciprocal-space points in which the Fock matrix is diagonalized. When $S=8$ is used, the energy difference with respect to the $S=4$ case is smaller than $10^{-5}$ Hartree/cell. As regards the basis set, Bloch functions are constructed from local functions ('atomic orbitals'), which in turn are linear combinations ('contractions') of gaussian-type functions (GTFs), and each of these is a product of a gaussian and a real solid spherical harmonic. The all-electron basis set used for the present study is very similar to that used in previous studies on $\mathrm{Cr}_{2} \mathrm{O}_{3}$ (with a $\mathrm{Cr}$ and $\mathrm{O}$ basis set) [6] and $\mathrm{MnO}$ (with a $\mathrm{Mn}$ basis set) [3], and contains 13 atomic orbitals for $\mathrm{O}$ and 27 for each of $\mathrm{Cr}$ and $\mathrm{Mn}$. The oxygen basis set can be denoted as an 8-411G contraction (the first shell is of s type and is a contraction of eight GTFs; then there are three sp shells). For $\mathrm{Cr}$ and $\mathrm{Mn}$, an 8-6-411-(41 d)G contraction is used, with two d-type shells. The exponents of the most diffuse sp and d shells of the three atoms have been optimized by searching for the minimum crystalline total energy. The best values $\left(\alpha_{\mathrm{sp}}(\mathrm{Mn})=1.21\right.$ and $0.50 ; \alpha_{\mathrm{sp}}(\mathrm{O})=0.49$ and $0.20 ; \alpha_{\mathrm{sp}}(\mathrm{Cr})=1.16$ and $\left.0.49 ; \alpha_{\mathrm{d}}(\mathrm{Cr})=0.34 ; \alpha_{\mathrm{d}}(\mathrm{Mn})=0.25\right)$ are very close to those optimized for $\mathrm{Cr}_{2} \mathrm{O}_{3}$ and $\mathrm{MnO}$.

\section{Results and discussion}

\subsection{Structure}

$\mathrm{MnCr}_{2} \mathrm{O}_{4}$ has the 'normal' (as opposed to 'inverse') spinel structure (space group $F d 3 m$ ), with two formula units in the unit cell (14 atoms). The $\mathrm{Cr}$ and $\mathrm{Mn}$ atoms are at $\left(\frac{1}{2}, \frac{1}{2}, \frac{1}{2}\right)$ 


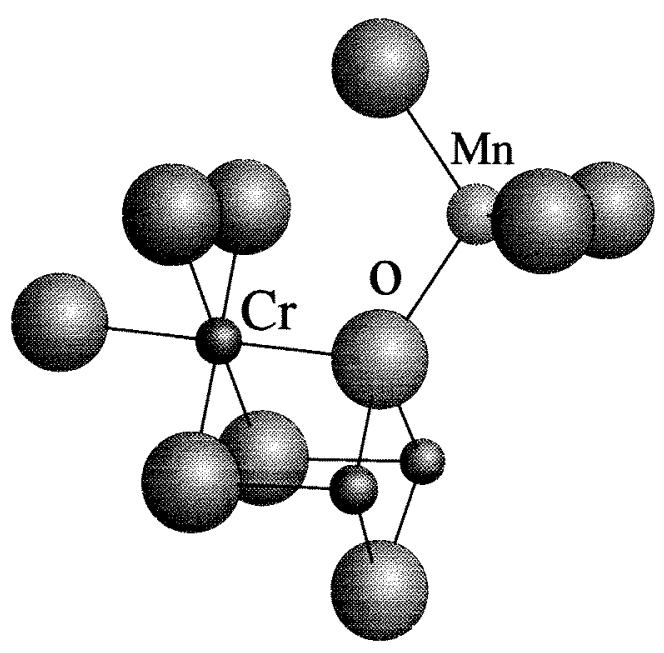

Figure 1. First-neighbour coordination for $\mathrm{Mn}, \mathrm{Cr}$ and $\mathrm{O}$ atoms.

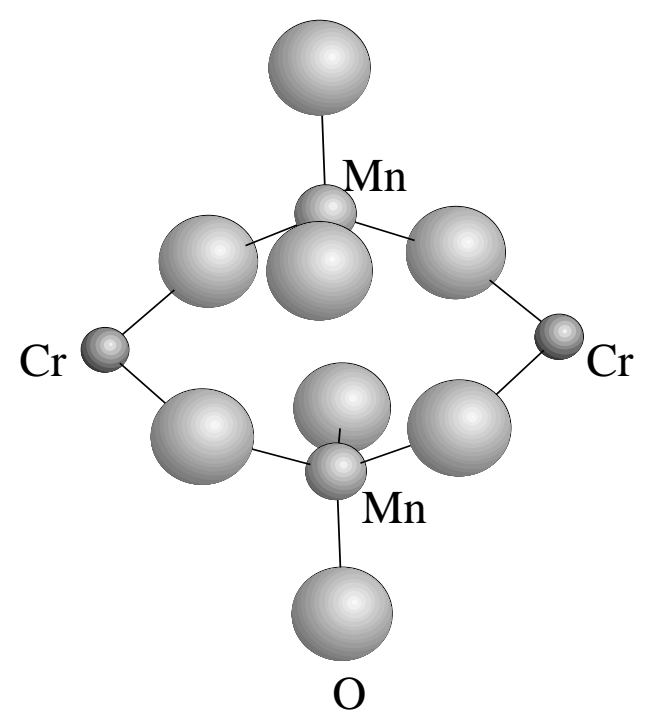

Figure 2. Paths for superexchange interactions.

and $\left(\frac{1}{4}, \frac{1}{4}, \frac{1}{4}\right)$ respectively, whereas $\mathrm{O}$ is at $(x, x, x)$. There are then only two geometrical parameters to optimize: the lattice parameter $a$ and the fractional coordinate of the oxygen atom, $x$. The nearest-neighbour coordination of $\mathrm{Cr}, \mathrm{Mn}$ and $\mathrm{O}$ is sketched in figure 1 (see page 68 of reference [11] for a figure showing the primitive cell). The $\mathrm{Cr}$ and $\mathrm{Mn}$ atoms occupy octahedral and tetrahedral sites, respectively. The $\mathrm{CrO}_{6}$ octahedron is regular only for the ideal geometry $\left(x=\frac{1}{4}\right)$, whereas the $\mathrm{MnO}_{4}$ tetrahedron is always regular. It is interesting to notice that each oxygen is fourfold coordinated with one $\mathrm{Mn}$ and three $\mathrm{Cr}$ nearest neighbours (figure 1). This is also clearly seen in figure 2, which will be used for the discussion of the superexchange interaction. As $x$ increases, the oxygen atoms are displaced along a [111] direction, causing the Mn tetrahedron to expand at the expense of 


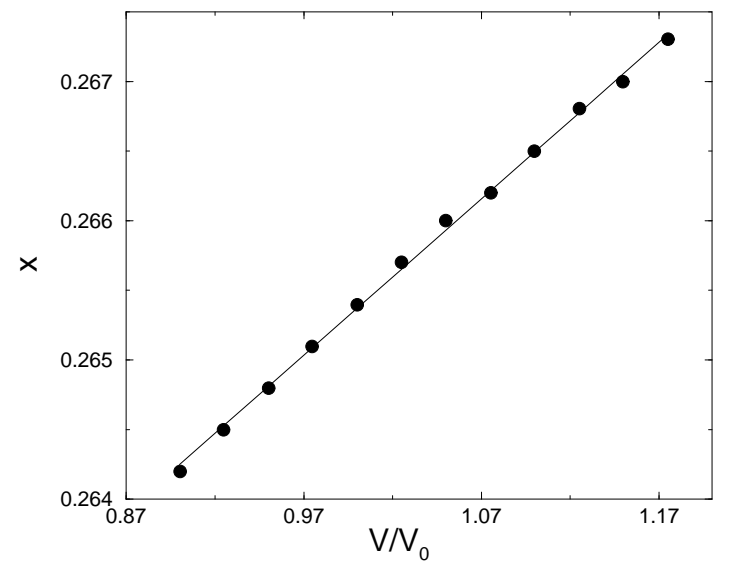

Figure 3. The fractional coordinate, $x$, as a function of the ratio $V / V_{0} \quad\left(V_{0}\right.$ is the experimental cell volume).

Table 1. The lattice parameter, $a(\AA)$, oxygen fractional coordinate, $x$, interatomic distances and angles (degrees) calculated for the equilibrium geometry. Experimental data (see reference [12]) are given for comparison.

\begin{tabular}{lllllllll}
\hline & $a$ & $x$ & $\mathrm{Mn}-\mathrm{O}$ & $\mathrm{Cr}-\mathrm{O}$ & $\mathrm{O}-\widehat{\mathrm{Cr}}-\mathrm{O}$ & $\mathrm{O}-\widehat{\mathrm{Mn}}-\mathrm{O}$ & $\mathrm{Cr}-\widehat{\mathrm{O}}-\mathrm{Mn}$ & $\mathrm{Cr}-\widehat{\mathrm{O}}-\mathrm{Cr}$ \\
\hline Experiment & 8.437 & 0.2641 & 2.03 & 2.00 & 83.0 & 109.5 & 120.4 & 89.7 \\
& & & & & 97.0 & & & \\
\hline Calculation & 8.582 & 0.2656 & 2.09 & 2.02 & 82.2 & 109.5 & 119.9 & 89.6 \\
& & & & & 97.8 & & & \\
\hline
\end{tabular}

the $\mathrm{Cr}$ octahedron, while the symmetry of the latter ( $m 3 m$ in the ideal case) downgrades to $3 m$. However, the $\mathrm{Cr}-\mathrm{O}$ bond distances remain the same. For the experimental geometry ( $a=8.437 \AA$ and $x=0.2641[12]$ ), the $\mathrm{Cr}-\mathrm{O}$ and $\mathrm{Mn}-\mathrm{O}$ distances are 2.00 and $2.03 \AA$, respectively; the $\mathrm{O}-\widehat{\mathrm{Cr}}-\mathrm{O}$ angles in the distorted octahedron are $83^{\circ}$ and $97^{\circ}$. The calculated equilibrium geometry of $\mathrm{MnCr}_{2} \mathrm{O}_{4}$ for the FEM solution has been obtained from a best fit with respect to the Murnaghan equation of state for 12 volumes $\left(0.9 V_{0}<V<1.175 V_{0}, V_{0}\right.$ being the experimental volume). At each volume, $x$ has been optimized (see figure 3). The resulting equilibrium lattice parameter is $8.582 \AA$, with an overestimation with respect to the experimental value of $2 \%$. The calculated fractional coordinate varies linearly with volume and its value at equilibrium $(x=0.2656)$ is in good agreement with experiment. The interatomic distances and angles deduced from the calculated equilibrium geometry are reported in table 1 . The angles are very close to the experimental values, as they depend on the $x$-value only. For the bulk modulus, $B$, a value of $208.6 \mathrm{GPa}$ has been obtained; we were unable to find an experimental value for comparison. The calculated bulk moduli for $\mathrm{Cr}_{2} \mathrm{O}_{3}$ and $\mathrm{MnO}$ are 262.5 and 164.0 GPa, respectively.

\subsection{Electronic behaviour}

All of the electronic properties were evaluated for the optimized geometry obtained for the ferromagnetic solution. The results are extremely similar for the ferromagnetic (FEM) and the two ferrimagnetic $\left(\mathrm{FIM}_{1}\right.$ and $\left.\mathrm{FIM}_{2}\right)$ solutions considered here, except the small differences in the spin density that will be discussed below.

The net charges resulting from a Mulliken analysis (see table 2) are $+1.85,+2.12$ and 
Table 2. Mulliken population data (in units of $|e|)$ : the electron charge $(\alpha+\beta)$ and net spin $(\alpha-\beta)$ for the atomic orbitals, and the overlap populations (X-O) for ferromagnetic $\mathrm{MnCr}_{2} \mathrm{O}_{4}$. The numbers in parentheses refer to the $\mathrm{FIM}_{1}$ or $\mathrm{FIM}_{2}$ solutions, and to oxygen atoms which are first neighbours of a $\mathrm{Mn} \downarrow$ ion.

\begin{tabular}{|c|c|c|c|c|c|c|c|}
\hline & & \multicolumn{2}{|c|}{$\mathrm{Cr}$} & \multicolumn{2}{|c|}{$\mathrm{Mn}$} & \multicolumn{2}{|r|}{$\mathrm{O}$} \\
\hline & & $\alpha+\beta$ & $\alpha-\beta$ & $\alpha+\beta$ & $\alpha-\beta$ & $\alpha+\beta$ & $\alpha-\beta$ \\
\hline Total & $\mathrm{sp}$ & 18.12 & 0.03 & 18.04 & 0.02 & 9.52 & $\begin{array}{l}-0.01 \\
(-0.05)\end{array}$ \\
\hline $\mathrm{d}\left(\mathrm{t}_{2 \mathrm{~g}}\right)$ & & 3.10 & 2.91 & 3.08 & 2.93 & & \\
\hline$d\left(e_{g}\right)$ & & 0.67 & 0.12 & 2.03 & 1.98 & & \\
\hline Total & $\mathrm{d}$ & 3.77 & 3.03 & 5.11 & 4.91 & & $\begin{array}{l}-0.01 \\
(-0.05)\end{array}$ \\
\hline Grand total & & 21.89 & 3.06 & 23.15 & 4.93 & 9.52 & \\
\hline Net charge & & +2.12 & & +1.85 & & -1.52 & \\
\hline $\mathrm{X}-\mathrm{O}$ & & 0.03 & -0.01 & -0.03 & -0.05 & -0.02 & 0.00 \\
\hline
\end{tabular}
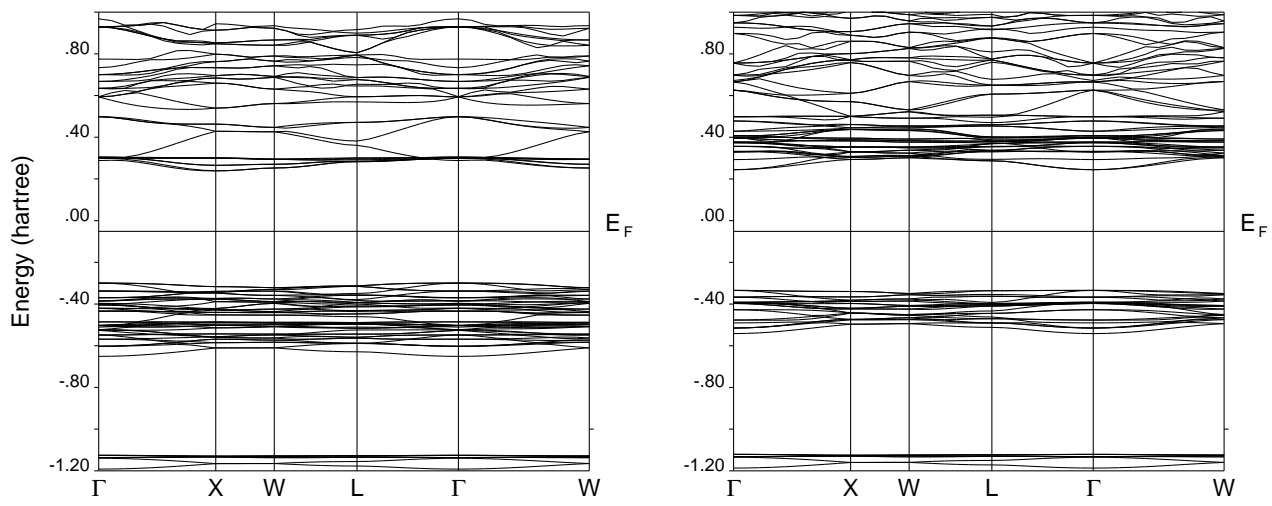

Figure 4. The $\alpha$ (left) and $\beta$ (right) band structure of ferromagnetic $\mathrm{MnCr}_{2} \mathrm{O}_{4} . E_{\mathrm{F}}$ is the Fermi level.

$-1.52|e|$ for $\mathrm{Mn}, \mathrm{Cr}$ and $\mathrm{O}$, respectively. By considering the ideal ionic state $\left(\mathrm{Mn}^{2+}, \mathrm{Cr}^{3+}\right.$ and $\mathrm{O}^{2-}$ ) as a reference, the most important modification is the charge transfer of about $0.5|e|$ from each nearest-neighbour oxygen atom to $\mathrm{Cr}$, whereas $\mathrm{Mn}$ is very close to the ideal situation. Most of the $0.9|e|$ transferred to $\mathrm{Cr}$ goes to the $\mathrm{d}$ shell in $\mathrm{e}_{\mathrm{g}}$ states, where both $\alpha$ - and $\beta$-states are populated ( 0.39 and $0.27|e|$, respectively). This indicates a certain degree of covalency, which is confirmed by the bond population data: $+0.03|e|$ for each of the six $\mathrm{Cr}-\mathrm{O}$ bonds (the same value is observed for $\mathrm{Cr}_{2} \mathrm{O}_{3}$ ), whereas the $\mathrm{Mn}-\mathrm{O}$ bond population is negative $(-0.03|e|)$ indicating a relatively strong repulsion between the two ions.

The band structures of $\mathrm{MnCr}_{2} \mathrm{O}_{4}$ for the $\alpha$ - and $\beta$-states of the FEM solution are shown in figure 4 ; the bands at about -1.2 Hartree are nearly pure oxygen $2 \mathrm{~s}$ bands; they are separated by a large gap from the upper valence bands, to which the $2 \mathrm{p}$ oxygen and the $3 \mathrm{~d}$ $\mathrm{Cr}$ and $\mathrm{Mn}$ electrons contribute. In the case of the $\beta$ band structure, only the $2 \mathrm{p}$ oxygen electrons contribute significantly, with minor contributions from $\mathrm{Cr} \mathrm{d}$ electrons $(0.37|e|)$. The gap between the valence and conduction bands is of 0.55 Hartree, confirming that $\mathrm{MnCr}_{2} \mathrm{O}_{4}$ is quite ionic. The projected density of states (DOS), figure 5, shows that the top 


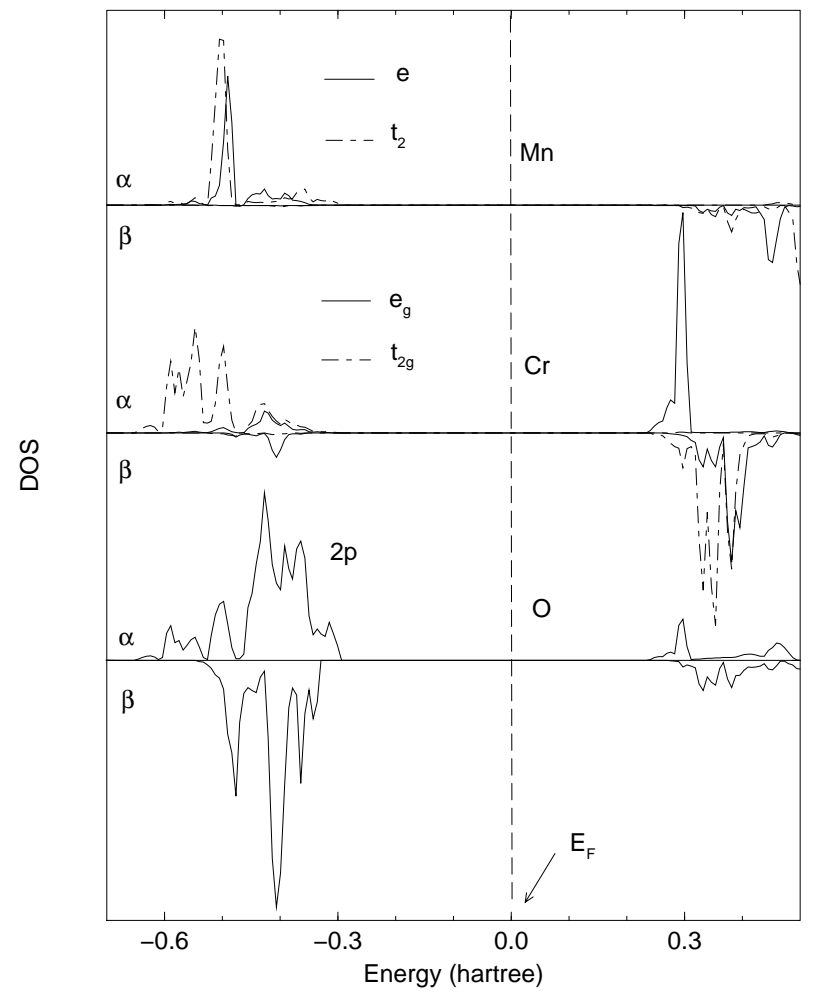

Figure 5. The density of electronic states (DOS) of $\mathrm{MnCr}_{2} \mathrm{O}_{4}$. Projections onto the atomic orbital contributions for the ferromagnetic solution are shown.

of the valence band is mainly contributed by $\mathrm{p}(\mathrm{O})$, whereas the bottom of the conduction band is given mainly by $d(\mathrm{Cr})$ : so, the energy gap has $\mathrm{p}-\mathrm{d}$ character, and $\mathrm{MnCr}_{2} \mathrm{O}_{4}$ appears to be an insulator with some charge-transfer character. The $\mathrm{Mnd}$ electrons give two sharp peaks at about -0.5 Hartree due to e and $t_{2}$ states containing nearly exactly five electrons (table 2), with only minor contributions to the valence bands above and below this value. Almost all of these electrons are in $\alpha$-states (5.01 $|e|)$; the very small contribution from $\beta$-states (about $0.1|e|$ ) is due to mixed terms including the contributions of the four nearest-neighbour oxygen atoms. The $\mathrm{Cr} \mathrm{d}$ states are, in contrast, more diffuse; the large band between -0.6 and -0.45 Hartree is due to $t_{2 g} \alpha$-electrons $(3.00|e|)$. The broad diffuse band between -0.45 and -0.30 Hartree, with contributions from both $\alpha$ and $\beta$ $\mathrm{e}_{\mathrm{g}}$ electrons $(0.40|e|$ and $0.27|e|$ respectively), overlaps with the diffuse oxygen $\mathrm{p}$ band, giving rise to a $\sigma$-bonding (upper valence bands) $\sigma$-antibonding (lower conduction bands) $\mathrm{Cr}-\mathrm{O}$ interaction. This feature, already observed for $\mathrm{Cr}_{2} \mathrm{O}_{3}$ (see figure 4 in reference [6]), indicates the presence of some covalent character in the $\mathrm{Cr}-\mathrm{O}$ bond, as has already been indicated by bond population data.

A map of the electron-density difference between the crystalline charge density and the superposition of the spherical ions $\mathrm{Mn}^{2+}, \mathrm{Cr}^{3+}$ and $\mathrm{O}^{2-}$ (whose wave-function has been obtained with the same basis set as was used for the bulk) is given in figure 6 . The usual spherical shrinking of the electron density of the cations and anions in the solid is observed; it is a consequence of the Madelung field and the exchange repulsion. A large redistribution of the Cr charge occurs in the solid, as, due to the crystal field, d levels split and only the 


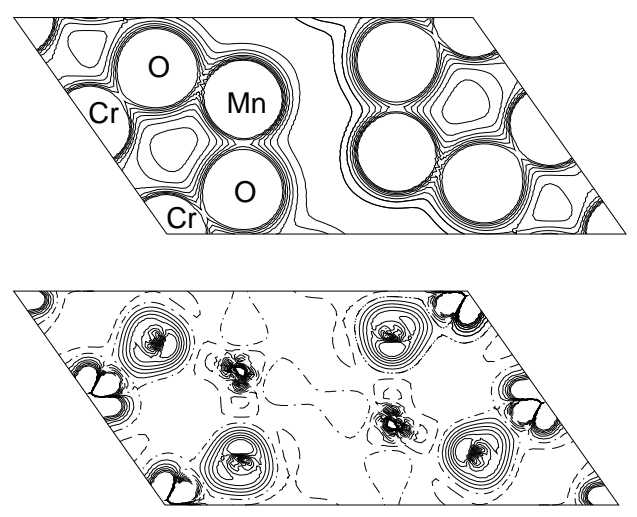

Figure 6. Total and difference (crystal minus ionic superposition) charge-density maps on a plane passing through $\mathrm{Mn}, \mathrm{O}$ and $\mathrm{Cr}$ atoms. The total and difference densities are truncated in the core region at 0.08 and $\pm 0.03 \mathrm{e} / \mathrm{Bohr}^{-3}$, respectively; the separations between two contiguous isodensity curves are 0.01 and $0.005 \mathrm{e} / \mathrm{bohr}^{-3}$, respectively. Continuous, chain and dashed lines in the difference map indicate positive, zero and negative values, respectively. Notice that, in the difference map, the function is negative in the central lobe of the $\mathrm{Cr}$ atom, the one directed towards the oxygen atom, and positive in the two lateral ones.

$\mathrm{t}_{2 \mathrm{~g}}$ set is occupied; a deformation of the oxygen charge towards the $\mathrm{Cr}$ ions is also observed in the direction of the hole (dashed lines for $\mathrm{Cr}$ ) created by filling the $\mathrm{t}_{2 \mathrm{~g}}$ orbitals. The modification of the charge distribution of $\mathrm{Mn}$ is less dramatic than for $\mathrm{Cr}$, because, in the solid, it retains the spherical symmetry of the isolated ion. The features shown by $\mathrm{Cr}$ and $\mathrm{O}$ in figure 6 are extremely similar to those shown by the same atoms in $\mathrm{Cr}_{2} \mathrm{O}_{3}$ (see figure 5 in reference [6]).

Table 3. The calculated equilibrium volume, $V$, and total energy per formula unit, $E$, for $\mathrm{MnO}$, $\alpha-\mathrm{Cr}_{2} \mathrm{O}_{3}$ and $\mathrm{MnCr}_{2} \mathrm{O}_{4}$, and the energy difference, $\Delta E$ (in kcal mol${ }^{-1}$ ), between $\mathrm{MnCr}_{2} \mathrm{O}_{4}$ and the mixture of oxides. HF $+\mathrm{d}(\mathrm{O})$ means that an optimized $\mathrm{d}$ shell has been added to the oxygen basis set. $\mathrm{HF}+\mathrm{P} 91+\mathrm{d}(\mathrm{O})$ refers to results including an a posteriori evaluation of the correlation energy through Perdew's functional [13].

\begin{tabular}{lcccccc}
\hline & & $\mathrm{MnO}$ & $\mathrm{Cr}_{2} \mathrm{O}_{3}$ & $\mathrm{MnO}+\mathrm{Cr}_{2} \mathrm{O}_{3}$ & $\mathrm{MnCr}_{2} \mathrm{O}_{4}$ & $\Delta E$ \\
\hline $\mathrm{HF}$ & $V\left(\AA^{3}\right)$ & 23.178 & 50.249 & 73.427 & 79.014 & \\
& $E(\mathrm{au})$ & -1224.8242 & -2311.5931 & -3536.4173 & -3536.4490 & 19.89 \\
\hline $\mathrm{HF}+\mathrm{d}(\mathrm{O})$ & $V\left(\AA^{3}\right)$ & 23.178 & 50.514 & 73.692 & 79.197 & \\
& $E(\mathrm{au})$ & -1224.8251 & -2311.6081 & -3536.4332 & -3536.4666 & 20.96 \\
\hline $\mathrm{HF}+\mathrm{P} 91$ & $V\left(\AA^{3}\right)$ & 20.993 & 46.813 & 67.806 & 73.423 & \\
$+\mathrm{d}(\mathrm{O})$ & $E(\mathrm{au})$ & -1226.3126 & -2314.7985 & -3541.1111 & -3541.1382 & 17.01 \\
\hline
\end{tabular}

\subsection{The equilibrium of the spinel decomposition}

The calculated equilibrium volumes and energies of $\mathrm{MnO}, \alpha-\mathrm{Cr}_{2} \mathrm{O}_{3}$ and $\mathrm{MnCr}_{2} \mathrm{O}_{4}$ are reported in table 3. At zero pressure and at the static limit, $\mathrm{MnCr}_{2} \mathrm{O}_{4}$ is more stable than the mixture of simple oxides by about $20 \mathrm{kcal} \mathrm{mol}^{-1}$ per formula unit at the HartreeFock level; basis set improvements, such as the addition of polarization $\mathrm{d}$ functions to 
oxygen, do not modify this number. As electron correlation effects are expected to play a non-negligible role, they have been estimated a posteriori (that is, by using the HartreeFock self-consistent charge density) with the correlation-only density functional proposed by Perdew [13]; the recalculated equilibrium volumes and energies are given in table 3 . The stability of the spinel with respect to the oxides decreases to $17 \mathrm{kcal} \mathrm{mol}^{-1}$. The $\mathrm{MnCr}_{2} \mathrm{O}_{4}$ experimental enthalpy of formation (see figure 4 of reference [8]) is $15 \mathrm{kcal} \mathrm{mol}^{-1}$. The corrections required for comparing the calculated and the experimental data $\left(H^{\exp }-H^{0}\right.$ and zero-point vibrational energies) are expected to increase the difference between the two numbers by no more than $2-3 \mathrm{kcal} \mathrm{mol}^{-1}$, so the agreement must be considered very satisfactory. These numbers are similar to those obtained in a previous study with the same method for the equilibrium $\mathrm{MgO}+\alpha-\mathrm{Al}_{2} \mathrm{O}_{3} \rightleftharpoons \mathrm{MgAl}_{2} \mathrm{O}_{4}\left(\Delta E=5.1 \mathrm{kcal} \mathrm{mol}^{-1}\right.$ at the HF-plus-correlation level, to be compared with $5.3 \mathrm{kcal} \mathrm{mol}^{-1}$ from experiment). Also, the equilibrium volume differences between the spinel and the mixture of oxides are very similar in the two cases ( $\Delta V$ is 5.59 and $4.94 \AA^{3}$ for $\mathrm{MnCr}_{2} \mathrm{O}_{4}$ and $\mathrm{MgAl}_{2} \mathrm{O}_{4}$, respectively). In both cases, the higher stability of the spinel at atmospheric pressure is due to a more efficient exploitation of the electrostatic interactions, only partially compensated by a higher short-range repulsion.

\subsection{Ferromagnetic and ferrimagnetic states}

In the present study, it was possible to investigate, together with the ferromagnetic solution (FEM, all spins up), two ferrimagnetic solutions, FIM $_{1}$ (where one Mn atom has opposite spin with respect to twelve $\mathrm{Cr}$ and four $\mathrm{Mn}$ second neighbours) and $\mathrm{FIM}_{2}$ (where all $\mathrm{Mn}$ atoms have opposite spin with respect to their own coordination sphere of twelve $\mathrm{Cr}$ atoms).

The superexchange paths for the $\mathrm{Cr} \uparrow-\mathrm{Mn} \downarrow$ (where the arrows indicate the spin associated to the metal ions) and $\mathrm{Mn} \uparrow-\mathrm{Mn} \downarrow$ interactions are shown in figure 2.

Table 4. Total energies per unit cell (in Hartree, for the experimental geometry) at the HartreeFock level $\left(E_{\mathrm{HF}}\right)$ and Hartree-Fock + a posteriori correlation-corrected level [13] $\left(E_{\mathrm{HF}+\mathrm{P} 91}\right)$. $\Delta E_{\mathrm{HF}}$ and $\Delta E_{\mathrm{HF}+\mathrm{P} 91}$ are the corresponding energy differences with respect to the ferromagnetic (FEM) solution (with all metal spins up). FIM 1 and FIM $_{2}$ indicate the two antiferromagnetic solutions (the former contains one $\mathrm{Mn} \uparrow$ (spin up) and one $\mathrm{Mn} \downarrow$ (spin down); the latter contains two $\mathrm{Mn} \downarrow$; in both phases all $\mathrm{Cr}$ are $\uparrow$ ).

\begin{tabular}{lllll}
\hline Solution & $E_{\mathrm{HF}}$ & $\Delta E_{\mathrm{HF}}$ & $E_{\mathrm{HF}+\mathrm{P} 91}$ & $\Delta E_{\mathrm{HF}+\mathrm{P} 91}$ \\
\hline FEM & -7072.88294 & - & -7082.22935 & - \\
FIM $_{1}$ & -7072.88478 & 0.00184 & -7082.23152 & 0.00217 \\
FIM $_{2}$ & -7072.88619 & 0.00325 & -7082.23317 & 0.00382 \\
\hline
\end{tabular}

Because of computational limitations, we were unable to explore the $\mathrm{Cr} \uparrow-\mathrm{Cr} \downarrow$ superexchange interaction; in fact, the point symmetry of a unit cell in which a fraction of $\mathrm{Cr}$ atoms possess $\alpha$-spin and the remaining atoms possess $\beta$-spin is very low, and the corresponding computational cost unmanageable at the moment. The total energies of the FEM, FIM ${ }_{1}$ and FIM $_{2}$ solutions are given in table 4. The energy differences $\Delta E\left(\mathrm{FEM}-\mathrm{FIM}_{1}\right)$ and $\Delta E\left(\mathrm{FEM}-\mathrm{FIM}_{2}\right)$ are measures of the $12 \mathrm{Cr} \uparrow-\mathrm{Mn} \downarrow+4 \mathrm{Mn} \uparrow-\mathrm{Mn} \downarrow$ and $24 \mathrm{Cr} \uparrow-\mathrm{Mn} \downarrow$ interactions, respectively. Under the hypothesis of additivity, it is possible to associate energies of 0.135 (0.159) and 0.054 (0.065) mHartree to each $\mathrm{Cr} \uparrow-\mathrm{Mn} \downarrow$ and $\mathrm{Mn} \uparrow-\mathrm{Mn} \downarrow$ interaction, respectively, at the HF (HF + P91 [13]) level. It is interesting to notice that the $\mathrm{Mn} \uparrow-\mathrm{Mn} \downarrow$ interaction is larger than expected on the basis of the 

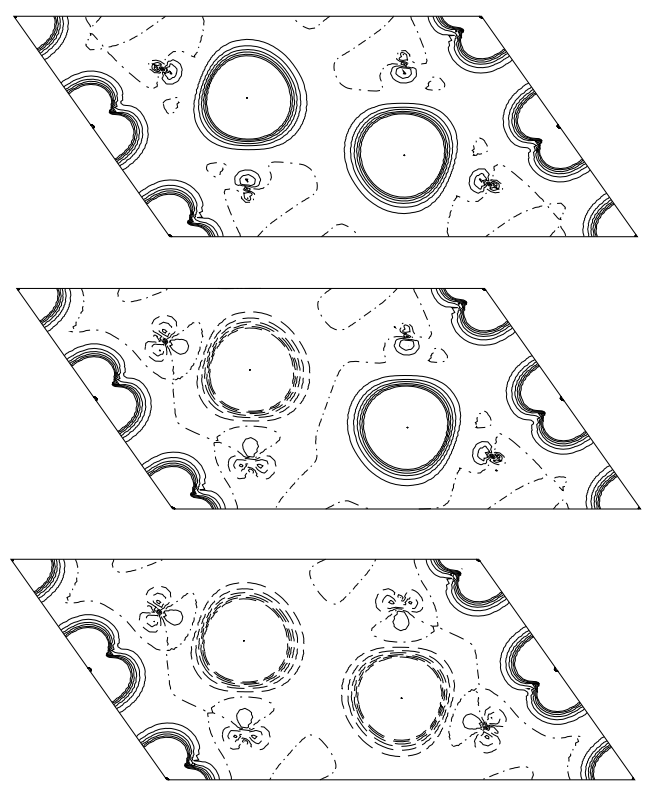

Figure 7. Spin-density maps of the FEM, FIM ${ }_{1}$ and $\mathrm{FIM}_{2}$ phases of $\mathrm{MnCr}_{2} \mathrm{O}_{4}$ in a plane passing through $\mathrm{Mn}, \mathrm{O}$ and $\mathrm{Cr}$ atoms. The plane is the same as in figure 6; the scale corresponds to that used for the difference map. Continuous and dashed lines correspond to an excess of $\alpha$ and $\beta$-electrons, respectively; chain lines correspond to equal values of the two densities.

superexchange path sketched in figure 2: the two atoms interact through a $\mathrm{Cr}$ atom and the corresponding bridging oxygen atoms. A direct $\mathrm{Mn} \uparrow-\mathrm{Mn} \downarrow$ interaction could be suggested by the relatively short distance ( $3.7 \AA$; see figures 6 and 7 ), but we did not find any trace of it in the population analysis data, or in the spin-density maps (figure 7). As regards the $\mathrm{Cr} \uparrow-\mathrm{Mn} \downarrow$ interaction, the usual interpretation scheme can be adopted, as in reference [14]: in the FEM state, the oxygen ion is surrounded by four metal ions whose external electrons have $\alpha$-spin; the short-range $\mathrm{Cr}-\mathrm{O}$ and $\mathrm{Mn}-\mathrm{O}$ repulsion then involves mainly the oxygen $\alpha$-electrons; as a consequence, there is a small spin polarization on the oxygen (see figure 7 and table 2). In the $\mathrm{FIM}_{2}$ state, the oxygen ions are surrounded by ions with $\alpha$ (three $\mathrm{Cr}$ ) and $\beta$ (one $\mathrm{Mn}$ ) d electrons; small displacements of the $\alpha$-or $\beta$-electrons on oxygen towards ions with opposite spin are sufficient to reduce the 'spin pressure' of the FEM solution. The total amount of charge involved in this movement is very small (less than $0.1|e|$; see table 2); one should remember, however, that the energy differences are very small too (about 4 mHartree; see table 4). The FIM $_{1}$ situation is intermediate between the FEM and FIM $_{2}$ cases. The spin polarization on the anion, and the mechanism connected to the stabilization of antiferromagnetic alignments are less evident here (see figure 7) than in other cases, where the anion is twofold coordinated with an $\mathrm{M}-\widehat{\mathrm{O}}-\mathrm{M}$ angle of $180^{\circ}$ [14]; the threefold-coordinated anion, as in rutile-type $\mathrm{MnF}_{2}$ structures [5, 6], is intermediate between the two previous types.

According to some authors, the spin ordering in $\mathrm{MnCr}_{2} \mathrm{O}_{4}$ is not of the ferrimagnetic collinear type discussed here, but involves more complicated spin orientations (spiral ordering; see figure 18 in reference [11]). However, limitations in both the Hamiltonian adopted (the UHF method provides spin-polarized states that are eigenfunctions of the spin operator $S_{z}$, but not of $S^{2}$ ) and the computational resources (some ferrimagnetic structures 
would require magnetic cells much larger than the non-magnetic one, with a substantial reduction of the point symmetry and subsequent increase in computational cost) prevent the exploration of these possibilities in the present work.

\section{Conclusions}

An ab initio quantum mechanical treatment has been successfully applied to the study of a relatively complicated crystalline structure, containing 14 atoms/cell, including six transition metal atoms (four $\mathrm{Cr}$ and two $\mathrm{Mn}$ ). The solid-state chemical reaction $\mathrm{MnO}+\alpha \mathrm{Cr}_{2} \mathrm{O}_{3} \rightleftharpoons \mathrm{MnCr}_{2} \mathrm{O}_{4}$ has been investigated; the results compare well with experiment and are of the same quality as those obtained for similar reactions between systems containing lighter atoms [11]. The electronic structure has been shown to be very close to the ideal ionic one for $\mathrm{Mn}$ (net charge $+1.85|e|, 5.11$ unpaired d electrons), whereas a relatively large $\mathrm{O} \rightarrow \mathrm{Cr}$ back-donation takes place with respect to the $\mathrm{O}^{2-}, \mathrm{Cr}^{3+}$ formal charges. Two ferrimagnetic states have been considered, which are more stable than

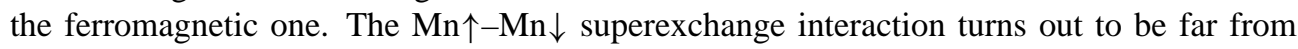
negligible, although, as expected, it is smaller than the $\mathrm{Cr} \uparrow-\mathrm{Mn} \downarrow$ one.

\section{Acknowledgments}

This work was supported by the Human Capital and Mobility Programme of the European Union under contract CHRX-CT93-0155, and by the the Italian MURST (40\%) and Italian CNR.

\section{References}

[1] Pisani C, Dovesi R and Roetti C 1988 Hartree-Fock ab initio Treatment of Crystalline Systems (Springer Lecture Notes in Chemistry 48) (Heidelberg: Springer)

[2] Saunders V R, Freyria-Fava C, Dovesi R, Salasco L and Roetti C 1992 Mol. Phys. 77629

[3] Mackrodt W C, Harrison N M, Saunders V R, Allan N L, Towler M D, Aprà E and Dovesi R 1993 Phil. Mag. 68653

[4] Towler M D, Allan N L, Harrison N M, Saunders V R, Mackrodt W C and Aprà E 1994 Phys. Rev. B 50 5041

[5] Catti M, Valerio G and Dovesi R 1995 Phys. Rev. B 517441

[6] Catti M, Sandrone G, Valerio G and Dovesi R 1996 J. Phys. Chem. Solids 571735

[7] Dovesi R, Saunders V R, Roetti C, Causà M, Harrison N M, Orlando R and Aprà E 1996 CRYSTAL95 User's Manual (Torino: University of Torino)

[8] Krupička S and Novák P 1982 Ferromagnetic Materials vol 3, ed E P Wohlfarth (Amsterdam: North-Holland) ch 4

[9] Catti M, Valerio G, Dovesi R and Causà M 1994 Phys. Rev. B 4914179

[10] Aprà E 1993 PhD Thesis University of Torino

[11] Adams D M 1974 Inorganic Solids. An Introduction to Concepts in Solid-State Structural Chemistry (London: Wiley)

[12] Raccah P M, Bouchard R J and Wold A 1966 J. Appl. Phys. 371436

[13] Perdew J P 1986 Phys. Rev. B 338822 (errata 198634 7406)

Perdew J P, Chevary J A, Vosko S H, Jackson K A, Pederson M R, Singh D J and Fiolhais C 1992 Phys. Rev. B 466671

[14] Ricart J M, Dovesi R, Roetti C and Saunders V R 1995 Phys. Rev. B 522381 (errata 199755 15942) 